\title{
Qualidade de vida e intensidade de dor em portadores de osteoporose
}

\section{Quality of life and intensity of pain on osteoporosis carriers}

\author{
Igor Andrade Barros'; José Luis Feltrin Oréficel; Rosalbina Santiago Rubint Oréficel; \\ Filipe Abdalla dos Reis ${ }^{1,2}$ \\ ${ }^{1}$ Curso de Fisioterapia da Universidade Anhanguera - Uniderp, Campo Grande -MS [Brasil]. \\ ${ }_{2}^{2}$ Programa de Pós-Graduação em Saúde e Desenvolvimento na Região Centro Oeste - UFMS, Campo Grande - MS [Brasil]. \\ Endereço para correspondência \\ Igor Andrade Barros \\ Rua Irmãos Vilasboas, 140, Vila Margarida \\ 79023-036 - Campo Grande, MS [Brasil] \\ ccfi@uniderp.br
}

\section{Resumo}

Objetivos: Avaliar a qualidade de vida (QV) e a intensidade da dor em sujeitos portadores de osteoporose. Métodos: Foram avaliados, por meio do questionário MOS SF-36 e Escala Visual Analógica de dor (EVA), doze sujeitos portadores de osteoporose. Para as variáveis descritas pelo questionário em relação à intensidade de dor, com nível de significância estabelecido em $\mathrm{p}<0,05$ foi utilizado o teste de correlação linear de Pearson. Resultados: Nos cruzamentos entre a EVA e os domínios do MOS SF-36, pode-se observar significância estatística para o estado geral de saúde, vitalidade e aspectos sociais. Observou-se baixo índice para a intensidade de dor $(3,1)$ e escore médio de 42 com classificação em boa QV. Conclusão: A QV dos portadores de osteoporose está classificada em boa e muito boa, e os entrevistados apresentaram uma intensidade de dor considerada baixa, sugestivo de não interferir na QV da população avaliada.

Descritores: Dor; Osteoporose; Qualidade de vida.

\begin{abstract}
Objectives: To assess the quality of life (QOL) and pain intensity in individuals with osteoporosis. Methods: We evaluated by the MOS SF-36 and Visual Analogue Scale (VAS), twelve subjects with osteoporosis. For the variables described in the questionnaire regarding the intensity of pain, with significance set at $p<0.05$ was used to test linear correlation of Pearson. Results: At the intersections between VAS and the domains of MOS SF-36, one can observe statistical significance for the general health, vitality and social aspects. We observed a low rate for pain intensity (3.1) and the average score of 42 ranked in good QOL. Conclusion: The QOL of patients with osteoporosis is classified as good or very good, and the interviewees showed a pain intensity was low, suggesting it would not affect the QOL of the population assessed.
\end{abstract}

Key words: Osteoporosis; Pain; Quality of life. 


\section{Introdução}

O envelhecimento pode ser conceituado como um processo dinâmico e progressivo, no qual a alterações morfológicas, funcionais e bioquímica vão limitando progressivamente o organismo, tornando-o mais susceptível às agressões intrínsecas e extrínsecas ${ }^{1}$.

Um dos fatores mais importantes no processo de envelhecimento é a associação de certas doenças com determinada faixa etária. Entre essa doenças, a osteoporose vem despertando um grande interesse em todo o mundo, pelo aumento de casos em pessoas com mais idade, causando então uma maior taxa de mortalidade e morbidade entre eles ${ }^{2}$.

Segundo Vieira ${ }^{3}$, o processo de formação, crescimento e desenvolvimento do tecido ósseo humano inicia-se durante a fase embrionária e segue até a idade adulta.

Além da função de sustentação e proteção estrutural, o esqueleto é um importante reservatório de bicarbonato e cálcio. Na deficiência desses minerais em nosso organismo eles são retirados de nossos ossos para fazer a reposição do nível normal que circula em nosso corpo².

A perda de massa óssea se dá de vários modos resultando na osteoporose, que é uma desordem esquelética crônica progressiva, de origem multifatorial, que acomete, na maioria dos casos, pessoas com mais idade e mulheres pós-menopausa ${ }^{2-3}$.

Defini-se a osteoporose como uma doença esquelética, sistêmica e metabólica que se caracteriza pela diminuição da massa óssea e deterioração da microarquitetura do tecido ósseo com susceptibilidade à fratura ${ }^{4,5}$.

A osteoporose caracteriza-se pela resistência óssea comprometida, predispondo o aumento de risco de fraturas, dor, deformidades e incapacidades físico-funcionais ${ }^{5,6}$.

Tanto a osteopenia quanto a osteoporose foram diagnosticadas, na população geral acima de 50 anos, em cerca de 44 milhões de pessoas, sendo 22 milhões mulheres osteopênicas e 8 milhões de osteoporóticas. As previsões para o ano de 2010 são de 26 e 9 milhões de mulheres osteopênicas e osteoporóticas, respectivamente 7 .

Predominantemente, a osteoporose acomete mais mulheres após menopausa e, em números menos expressivos, em idosos do sexo masculino e, segundo Rennó, Driusso, Ferreira ${ }^{1}$, ela afeta um terço das mulheres após menopausa.

Estima-se que uma em cada duas mulheres e um em cada cinco homens acima de 65 anos apresentarão, em algum momento da vida, pelo menos uma fratura relacionada a essa patologia. A osteoporose encontra-se entre as maiores causas de morbidade entre os idosos, e sua incidência aumenta, acompanhando a tendência demográfica etária da população mundial e seus respectivos aspectos na expectativa de vida ${ }^{1}$.

Sua etiologia está baseada em distúrbios hormonais após menopausa, na alimentação pobre de minerais do tipo cálcio e de vitaminas, essenciais para um bom funcionamento do organismo, associado ao tempo de imobilização prolongado em idosos e pela falta de descarga de peso para uma maior produção de estímulos aos osteoblastos ${ }^{8}$.

A osteoporose pode ser classificada quanto a sua origem, sendo a do tipo I, também conhecida por osteoporose pós-menopausa, apresenta perda óssea acelerada, sendo mais comum em mulheres, atinge predominantemente o osso trabecular e é considerada uma das etiologias das fraturas de vértebras e de rádio distal ${ }^{6}$.

Já a Osteoporose do tipo II, ou senil, é relacionada ao envelhecimento e aparece por deficiência crônica de cálcio, aumento da atividade do paratormônio, por diminuição das funções das glândulas adrenais, hipertiroidismo secundário e diminuição da formação óssea. Ocorre perda proporcional de osso cortical e trabecular, em contraste com a perda desproporcional de osso trabecular do tipo I. A osteoporose do tipo II está associada a fraturas de quadril, colo de fêmur, tíbia e pelves em mulheres idosas e homens com mais de 65 anos ${ }^{7}$. 
Com relação à osteoporose secundária ela é decorrente de processos inflamatório, como artrite reumatoide; ou por mieloma múltiplo; por falta de exercícios físicos; por uso abusivo do álcool ou medicamentos contendo heparina, vitamina A e corticoides. Os corticoides inibem a reabsorção intestinal do cálcio e aumentam sua eliminação urinária, diminuem a formação osteoblástica e aumentam a reabsorção osteoclástica ${ }^{7}$.

A osteoporose é um problema cínico e social de relevância, com sérias consequências clínicas. A mais frequente é a dor, que pode ter grande impacto no desenvolvimento das atividades cotidianas, influenciando no bem-estar e na qualidade de vida relacionada à saúde. Frequentemente os portadores de osteoporose apresentam limitações, para exercer normalmente suas atividades profissionais, sociais e de lazer, e um comprometimento emocional importante, que resulta na perda de sua qualidade de vida ${ }^{8}$.

O questionário The Medical Outcomes Study 36-item Short-Form Health Survey (SF-36) é um instrumento genérico que foi desenvolvido nos Estados Unidos para mensurar oito dos conceitos mais relacionados à qualidade de vida. $\mathrm{O}$ questionário idealizado por Ware e Sherbourne (1992), traduzido e validado para português por Ciconelli (1997), aplica-se, nas condições gerais relacionadas a patologias, à população de modo geral e específica, e se utiliza em estudos de caráter quantitativo e qualitativo ${ }^{8-9-10}$.

Com relação à dor, vários são os instrumentos de avaliação existentes, entre eles, a Escala Visual Analógica de Dor (EVA), que consiste em uma linha de $10 \mathrm{~cm}$, com âncoras verbais de "ausência de dor" à esquerda e "a pior dor possível" à direita, sendo orientada no plano horizontal, onde o paciente irá assinalar com um traço o que corresponde a sua ausência de dor ou a sua pior dor possível, no entanto, é considerado um instrumento unidimensional para avaliação da dor ${ }^{10}$.

Assim esse estudo teve por objetivo avaliar a qualidade de vida e a intensidade da dor em Portadores de Osteoporose submeti- dos a tratamento fisioterapêutico no Centro de Reabilitação da Universidade Anhanguera - Uniderp, na cidade de Campo Grande - MS.

\section{Casuística e métodos}

\section{Participantes e local de desenvolvimento da pesquisa}

Foram avaliados 12 sujeitos portadores de osteoporose em tratamento fisioterapêutico no Centro de Reabilitação da Universidade Anhanguera - Uniderp, sendo 10 do sexo feminino 2 do sexo masculino. Os indivíduos apresentaram média de idade de $71,9 \pm 5,8$ anos com $2(16,7 \%)$ do sexo masculino e $10(83,3 \%)$ do sexo feminino.

\section{Termo de consentimento e período realizado da pesquisa}

Os participantes da pesquisa assinaram a um termo de consentimento livre e esclarecido que obedece às normas de pesquisa para seres humanos de acordo com a resolução 196/96, de modo voluntário e sem nenhum ônus financeiro.

A referida pesquisa foi realizada nos meses de janeiro a abril de 2007.

\section{Critérios de inclusão e de exclusão para a pesquisa}

Como critérios de inclusão, foram utilizados sujeitos portadores de osteoporose, diagnosticada clinicamente por meio do exame de densitometria óssea, em tratamento fisioterapêutico no Centro de Reabilitação da Universidade Anhanguera - Uniderp.

Foram excluídos da pesquisa os indivíduos portadores de osteoporose, com alterações cognitivas, sem diagnóstico clínico, com comprometimento nervoso periférico, com osteopenia, que não esteja em tratamento no referido Centro de Reabilitação. 


\section{Material utilizado}

O questionário genérico de avaliação de qualidade de vida, é um instrumento tanto autoadministrável quanto administrável também por terceiros, composto por oito conceitos sobre a saúde: funcionamento do organismo, dor corporal, socialização, saúde mental, vitalidade, percepção geral da saúde, problemas físicos e emocionais. Cada um desses conceitos possui um número variável de itens que graduam as respostas e, após essas graduações, são recalculadas para então serem demonstradas em um score que varia de 0-100 pontos. A pontuação 0 (zero) representa estado de saúde ruim e a pontuação 100 (cem) representa excelente estado de saúde.

Visando classificar os escores dos domínios avaliados pelo instrumento de QV foi utilizado pelo pesquisador uma tabela de equivalência para a determinação mais clara da QV, segundo Oréfice ${ }^{12}$.

Essa tabela foi idealizada tendo como base a forma como se calcula os escores do questionário de QV. O MOS SF-36 tem seus dados divididos por 100 para alcançar os resultados finais, assim tem-se o percentual relativo a cada domínio avaliado ${ }^{12}$.

A tabela 1 distribui os escores de zero a cem divididos em cinco grupos que possuem, além dos dados numéricos, descritores para classificação dos domínios avaliados nos questionários ${ }^{12}$.

Tanto para a coleta quanto para análise da avaliação da dor nos participantes, utilizou-se a Escala Visual Analógica de Dor (EVA), idealizada por Scott e Huskisson em 1976, e reproduzida por Chapman e Syrjala em 1990.

Tabela l: Classificação da qualidade de vida

\begin{tabular}{c|c}
\hline Escore & Classificação \\
\hline 0 a 20 & Muito ruim estado de saúde / QV. \\
\hline 21 a 40 & Ruim estado de saúde / QV. \\
\hline 41 a 60 & Bom estado de saúde / QV. \\
\hline 61 a 80 & Muito bom estado de saúde / QV. \\
\hline 81 a 100 & Excelente estado de saúde / QV. \\
\hline
\end{tabular}

Essa escala consiste em uma linha de $10 \mathrm{~cm}$ com âncoras verbais de "ausência de dor" à esquerda e "a pior dor possível" à direita, quando é orientada no plano horizontal, ou ainda, seguindo a mesma temática só que disposta na vertical, onde a linha de $10 \mathrm{~cm}$ fica similar a um termômetro, o paciente irá assinalar com um traço o que corresponde a sua ausência de dor ou a sua pior dor possível, sendo a parte alta correspondente "a pior dor possível" e a parte baixa da linha correspondendo a "ausência de dor", no entanto, é considerado um instrumento unidimensional para avaliação da dor. Após a demarcação feita pelos participantes da pesquisa, uma régua de $10 \mathrm{~cm}$ serviu para graduar os valores numéricos e, posteriormente, tabulá-los e interpretá-los ${ }^{13}$.

Para a coleta e análise da dor e de sua localização, foi utilizado um diagrama corporal respectivamente, sendo este último extraído do questionário de Dor de McGill (Figura 1).

O questionário de dor de McGill, em sua forma abreviada, é provavelmente o mais conhecido dos instrumentos multidimensionais para mensuração da dor. Traduzido e validado para a língua portuguesa por Teixeira e Pimenta em 1997, esse

\section{Possui algum tipo de Dor Corporal? \\ ( ) Sim ( ) Não}

Onde sua dor se localiza?

Por favor, marque nos desenhos abaixo, as áreas em que você sente dor. Use E se externa, I se interna, próximo às áreas marcadas. Use El se ambas.

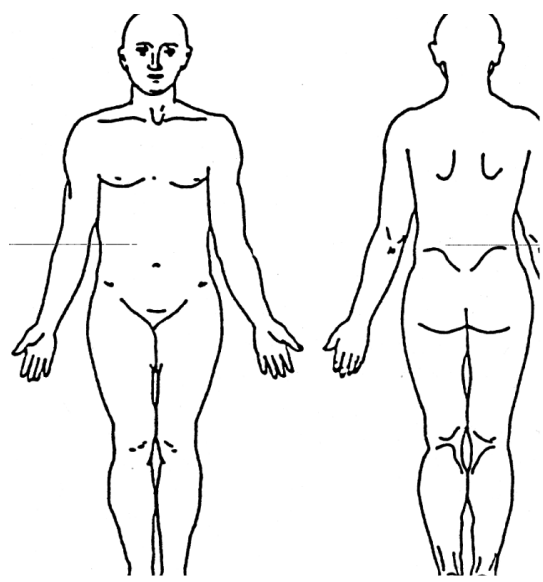

Figura 1: Diagrama corporal 
questionário possui como parte integrante, um diagrama corporal para as informações relacionadas com a localização anatômica da dor, e o entrevistado marca o local da dor com as iniciais I para dor interna, E para dor externa e EI para ambas ${ }^{14}$.

\section{Anớlise estatística}

Os dados foram tabulados por Microsoft Excel 2003 e analisados estatisticamente no software GraphPad Prism 4.0. O teste utilizado foi o de coeficiente de correlação linear de Pearson e o nível de significância estabelecido em $\mathrm{p}<0,05$.

Para a análise estatística foi realizado teste para verificação da hipótese de correlação entre qualidade de vida e avaliação da dor.

\section{Resultados}

Os resultados do estudo feito com relação aos domínios do SF - 36 estão descritos nas tabela 2 e figura 2 que mostram as médias dos seguintes domínios: capacidade funcional, aspec- tos físicos, dor, estado geral de saúde, vitalidade, aspectos sociais, emocionais e saúde mental.

Quando avaliados em relação à dor todos relataram possuir alguma sensação ou área corporal dolorosa, onze $(91,7 \%)$ disseram que sentiam a dor do tipo I - interna e um $(9,3 \%)$ afirmou que sentia a dor do tipo EI - externa e interna. Os sujeitos descreveram, em média, $3 \pm 1$ áreas de dor corporal e também um valor médio

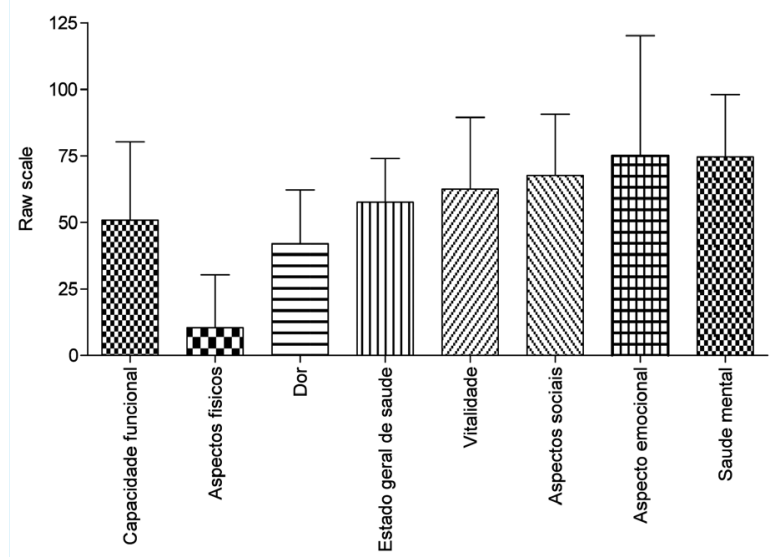

Figura 2: Valores médios \pm dp do raw scale do questionário genérico de avaliação da qualidade de vida (MOS SF - 36). (N=12)

Tabela 2: Valores do raw scale da aplicação do questionório genérico de avaliação da qualidade de vida (MOS SF - 36)

\begin{tabular}{c|c|c|c|c|c|c|c|c}
\hline $\mathrm{N}$ & $\begin{array}{c}\text { Capacidade } \\
\text { funcional }\end{array}$ & $\begin{array}{c}\text { Aspectos } \\
\text { físicos }\end{array}$ & Dor & $\begin{array}{c}\text { Estado } \\
\text { geral de } \\
\text { saúde }\end{array}$ & Vitalidade & $\begin{array}{c}\text { Aspectos } \\
\text { sociais }\end{array}$ & $\begin{array}{c}\text { Aspecto } \\
\text { emocional }\end{array}$ & $\begin{array}{c}\text { Saúde } \\
\text { mental }\end{array}$ \\
\hline 1 & 90,0 & 25,0 & 52,0 & 72,0 & 95,0 & 100,0 & 100,0 & 100,0 \\
\hline 2 & 15,0 & 0,0 & 31,0 & 35,0 & 30,0 & 62,5 & 0,0 & 44,0 \\
\hline 3 & 20,0 & 0,0 & 54,0 & 67,0 & 45,0 & 62,5 & 100,0 & 84,0 \\
\hline 4 & 60,0 & 50,0 & 52,0 & 85,0 & 90,0 & 100,0 & 100,0 & 96,0 \\
\hline 5 & 85,0 & 0,0 & 84,0 & 72,0 & 95,0 & 100,0 & 0,0 & 88,0 \\
\hline 6 & 100,0 & 50,0 & 22,0 & 45,0 & 45,0 & 50,0 & 0,0 & 32,0 \\
\hline 7 & 60,0 & 0,0 & 62,0 & 55,0 & 50,0 & 37,5 & 100,0 & 44,0 \\
\hline 8 & 50,0 & 0,0 & 42,0 & 62,0 & 80,0 & 50,0 & 100,0 & 64,0 \\
\hline 9 & 50,0 & 0,0 & 41,0 & 67,0 & 85,0 & 87,5 & 100,0 & 100,0 \\
\hline 10 & 30,0 & 0,0 & 12,0 & 30,0 & 20,0 & 50,0 & 100,0 & 80,0 \\
\hline 11 & 15,0 & 0,0 & 21,0 & 45,0 & 75,0 & 50,0 & 100,0 & 76,0 \\
\hline 12 & 35,0 & 0,0 & 31,0 & 57,0 & 40,0 & 62,5 & 100,0 & 88,0 \\
\hline Média & 50,8 & 10,4 & 42,0 & 57,7 & 62,5 & 67,7 & 75,0 & 74,7 \\
\hline DP & $\pm 29,4$ & $\pm 19,8$ & $\pm 20,2$ & $\pm 16,4$ & $\pm 26,9$ & $\pm 22,9$ & $\pm 45,2$ & \pm 23, \\
\hline
\end{tabular}


de 3,1 $\pm 2,1$ para a intensidade dessa dor avaliada pela Escala Visual Analógica de Dor (EVAD).

Ao aplicar o teste de correlação linear de Pearson entre a Escala Visual Analógica de Dor e a Qualidade de Vida entre os domínios do MOS SF -36 , obtivemos os resultados descritos na tabela 3.

Para os cruzamentos entre a EVA e os domínios estado geral de saúde, vitalidade, aspectos sociais, a correlação foi estatisticamente significativa, conforme apresentado nas figuras 3.

Tabela 3: Coeficiente de correlação linear de Pearson para a avaliação da escala visual de dor e os domínios do questionório $\mathrm{SF}-36 .(\mathrm{N}=12)$

\begin{tabular}{c|c|c}
\hline EVAD & Valor de Pearson $r$ & Valor de $p$ \\
\hline CF & $-0,562$ & 0,0572 \\
\hline AF & $-0,3924$ & 0,207 \\
\hline Dor & $-0,4415$ & 0,1508 \\
\hline EGS & $-0,7066$ & ${ }^{*} 0,0102$ \\
\hline Vit & $-0,5805$ & ${ }^{*} 0,0478$ \\
\hline AS & $-0,7889$ & ${ }^{*} 0,0023$ \\
\hline AE & $-0,0524$ & 0,8714 \\
\hline SM & $-0,5038$ & 0,095
\end{tabular}

Nota: EVAD - Escala Visual Analógica de Dor; $\mathrm{CF}$ - capacidade funcional, $\mathrm{AF}$ - aspectos físicos, EGS - estado geral de saúde, Vit - Vitalidade, AS - aspectos sociais, AE - aspecto emocional, $\mathrm{SM}$ - saúde mental.

$\left.{ }^{*}\right)$ correlação negativa estatisticamente significante.

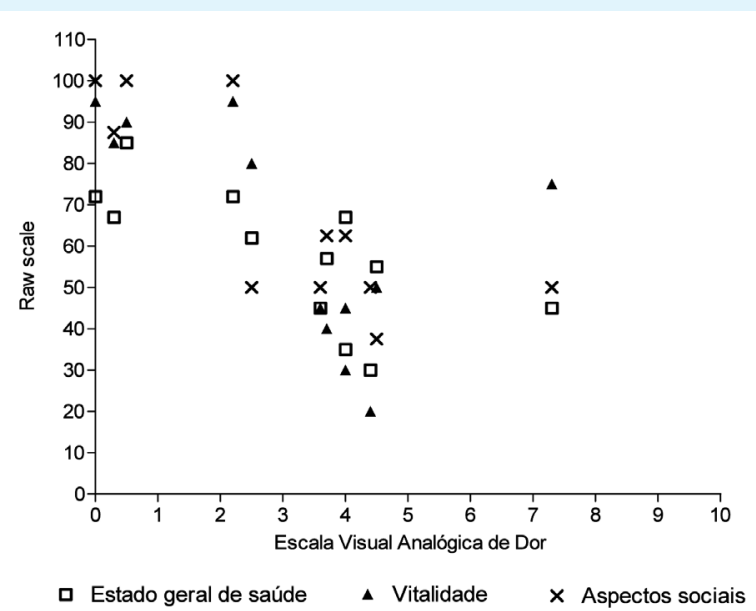

Figura 3: Coeficiente de correlação linear de Pearson para as comparações entre a EVA e os domínios do Questionário SF - 36: Estado Geral de Saúde, Vitalidade e Aspectos sociais

\section{Discussão}

No que se refere a faixa etária, esse estudo tem relação positiva com o trabalho de Aranha et al. ${ }^{9}$ quando avalia a qualidade de vida (QV) de idosas, relacionando-a com a saúde das portadoras de Osteoporose da população espanhola. Verifica-se que ambos obtiveram média de idade maior que 65 anos, assim como Lemos et al. ${ }^{15}$ que em seu estudo com idosos portadores de osteoporose avaliados em domicílio com o MOS SF - 36 apresentaram faixa etária maior que 60 anos.

Isso pode ser explicado pelo fato de ser a osteoporose pós-menopausa ou do tipo I, pois a queda na produção dos estrogênios, característica do estágio de vida após a menopausa, é um fator que acelera a redução da Densidade de Massa Óssea (DMO), colocando as mulheres como uma população especialmente suscetível. Além disso, a osteoporose primária do tipo I - ou após a menopausa, também tem despertado um grande interesse da saúde pública, pois no Brasil, $10 \%$ da população feminina vive um terço ou mais do total de anos de vida após a menopausa e a meta de assistência a essa parcela inclui medidas com enfoque na mudança comportamental e em investimento de longo prazo na saúde ${ }^{16}$.

Quanto ao sexo, foram avaliados, nesse trabalho, 10 pacientes do sexo feminino e 2 do sexo masculino. Já no trabalho de Aranha et al. ${ }^{9}$ foram 60 pacientes do sexo feminino e nenhum do sexo masculino, e no trabalho de Lemos et al. ${ }^{15}, 40$ pacientes do sexo feminino e nenhum do sexo masculino, fato este explicado por Rennó, Driusso, Ferreira ${ }^{1}$ e Jovine et al. $^{16}$, que relatam ser a osteoporose predominante em mulheres após a menopausa e, em números menos expressivos, em homens idosos.

A osteoporose afeta um terço das mulheres pós-menopausa, pois há queda na produção dos estrogênios, característica do estágio de vida após a menopausa, e estima-se ainda que, uma em cada duas mulheres e um em cada cinco homens acima de 65 anos apresentarão, em algum momento da vida, pelo menos uma fratura relacionada a esta condição clínica descrita ${ }^{1,16}$. 
Os sujeitos apresentam bom estado de saúde em relação à QV, para os aspectos emocionais avaliados pelo questionário. Já no estudo de Aranha et al. ${ }^{9}$ verificou-se um escore de vitalidade de 53.7, já Lemos et al. ${ }^{15}$ apresentaram um escore de 57.75. Comparando com os resultados presentes, observa-se que foram mais elevados, pois os pacientes entrevistados estavam sendo submetidos a tratamento fisioterápico no centro de reabilitação da Universidade Anhanguera - Uniderp.

Segundo relatos literários descritos para a intervenção nesses casos, as medidas a serem adotadas para modificações da QV, são fundamentalmente baseadas nas práticas de exercícios físicos e terapêuticos para que consigam reduzir a sintomatologia clínica referida pelos portadores de osteoporose ${ }^{17}$.

Quanto aos aspectos sociais Aranha et al. ${ }^{9}$ apresentam um escore de 89.0 enquanto Lemos et al. ${ }^{15}$, um escore de 71.56, ambos os estudos obtiveram valores mais elevados que esse estudo. Para explicar essa diferença cita-se os escores dos aspectos físicos, onde Aranha et al. ${ }^{9}$ traz um escore de 59.9 enquanto o trabalho de Lemos et al. ${ }^{15}$ têm escore de 42.0 para os aspectos físicos, valores esses superiores aos desse estudo, o que indica que os aspectos sociais podem ser melhorados pela realização de atividades tanto físicas quanto terapêuticas, que serviriam especificamente para melhora dos aspectos físicos, auxiliariam na socialização, uma vez que são realizadas em espaços específicos com número maior de pessoas, e possibilitam que as experiências sejam compartilhadas ${ }^{18}$.

Para os escores relativos aos aspectos emocionais, o trabalho de Aranha et al. ${ }^{9}$ apresenta um escore de 72.2, enquanto o trabalho de Lemos et al. ${ }^{15}$ indica um escore de 45.81 considerado muito ruim em relação ao estudo em questão.

Pode-se explicar esses efeitos com os achados descritos por Cantarelli et al. ${ }^{18}$ que descrevem que portadores de osteoporose têm sequelas psicológicas, como ansiedade, depressão, perda de autoestima e até isolamento social, podendo explicar também o escore da saúde men- tal dos avaliados por Aranha et al. ${ }^{9}$ que possuem escores de 63.0 e um escore de 65.0 para Lemos et al. ${ }^{15}$.

Nos aspectos relacionados com a capacidade funcional, Aranha et al. ${ }^{9}$ apresentam escore de 47.7, enquanto Lemos et al. ${ }^{15}$ mostram escores de 55.75, dados esses diferentes dos encontrados no trabalho realizado, pois são maiores que o de Aranha et al. ${ }^{9}$, porém, menores que os valores de Lemos et al. ${ }^{15}$, que relatam que os portadores de osteoporose têm que apresentar uma boa mobilidade para executar seu trabalho no dia a dia sem restrições e sintomatologias dolorosas, por exemplo, sendo assim mais independentes ${ }^{18}$.

Em relação ao estado geral de saúde não houve uma variação de resultado muito significativa, Aranha et al. ${ }^{9}$ trazem um escore de 45.1 e Lemos et al. ${ }^{15}$ mostram um escore de 65.92. Verifica-se que o valor do escore desse estudo ficou entre os valores citados, o que pode indicar que não houve alteração entre as populações pesquisadas. Diante disso, podese dizer que, ao analisar os resultados, que a QV dos participantes desse estudo apresentou melhores resultados se comparados aos obtidos por Aranha et al. ${ }^{8}$ e Lemos et al..$^{15}$, pois os pacientes estavam sendo submetidos a tratamento fisioterápico no centro de reabilitação da Universidade Anhanguera - Uniderp.

Com relação ao domínio Dor, os participantes do estudo de Aranha et al. ${ }^{9}$ apresentaram um escore de 52.3, já no estudo de Lemos et al. $^{15}$, o escore foi de 57.37, enquanto o escore desse estudo ficou abaixo destes, ou seja, os participantes desse estudo sentem mais dor quando comparados com os estudos descritos.

No entanto, vale ressaltar que quando se observa os dados da aplicação da EVA com valores de 3.1 \pm 2.1 que é considerado um valor baixo para a dor, pode-se questionar os achados do MOS SF - 36, podendo considerar como fator dessa diferença, em relação a avaliação da dor, os questionamentos do instrumento que se reportam as últimas quatro semanas, enquanto para a aplicação da EVA os dados eram antes e após a realização da intervenção fisioterapêutica. 
Outros dados descritos na literatura como, por exemplo, Jovine et al. ${ }^{16}$ podem servir para explicar as diferenças, porém colocando o questionário utilizado em dúvida quanto a sua sensibilidade para mensurar esses achados em portadores de osteoporose, embora trata-se de um instrumento genérico de avaliação da $\mathrm{QV}^{17}$.

Acredita-se que o treinamento resistido pode ser capaz de promover estímulos para aumentar a força muscular e a formação óssea, influenciando nos fatores de risco relacionados com a osteoporose $\mathrm{e}^{16,18,19}$.

Essas diferenças ficaram explicitas também quando comparadas por meio da correlação linear de Pearson que não mostrou correlação significativa $(p=0,0150)$ entre a dor avaliada pelo instrumento MOS SF - 36 em relação à EVA, podendo sugerir que o tratamento realizado tenha surtido efeito momentâneo e que, em longo prazo, não estejam suprindo as expectativas do tratamento.

Os resultados desse estudo revelam que os sujeitos atendidos no Centro de Reabilitação apresentam uma qualidade de vida classificada como muito boa e boa, segundo critérios estabelecidos neste estudo, dados estes que corroboram com Aranha et al. ${ }^{9}$, pois seus escores indicam muito boa e boa QV e também não diferenciam dos resultados de Lemos et al. ${ }^{15}$ para a QV dos seus entrevistados.

Esses resultados não são condizentes com os aspectos clínicos ${ }^{20}$ da doença e com a história natural da enfermidade osteoporótica que sempre apontam para uma ruim $\mathrm{QV}^{8}$.

\section{Conclusão}

Conclui-se que a qualidade de vida dos sujeitos portadores de osteoporose atendidos no Centro de Reabilitação da Universidade Anhanguera - Uniderp está classificada como boa e muito boa, e que só houve correlação significativa entre três domínios do MOS SF - 36 em relação a intensidade de dor avaliada por meio da EVA, o que pode sugerir a necessidade de se alterar a QV do grupo avaliado.

\section{Referências}

1. Rennó ACM, Driusso P, Ferreira V. Atividade física e osteoporose. Fisiot Mov. 2001 març; 13(2):76-82.

2. Neto PMA, Soares A, Urbantes AA, Araujo C A, Ferrari MEA; Amaral B, Moreira C, et al. Consenso brasileiro de osteoporose. Rev Bras Reumat.2002 dez;42(6):112-17.

3. Vieira RA, D'alessandro CC, Reis EDS, Paiva JP, Xavier KL, Rodriges LA, Barbalho MC, Aquino MO, Maria PC, Ribeiro VML. A atuação da fisioterapia na prevenção de quedas em pacientes com osteoporose senil. Fisiot Brasil.2002 abr;3(2):72-8.

4. Narciso BV, Azevedo E. Osteoporose em homens. Reumat Brasil. 2004 jun;5(2):55-8.

5. Bennell KL, Matthews B, Greig A, Briggs A, Kelly A, Sherburn M, Larsen J, Wark J. et al. Effects of an exercise and manual therapy program on physical impairments, function and quality-of-life in people with osteoporotic vertebral fracture: a randomised, single-blind controlled pilot trial. BMC Musculoskelet Disord.2010 feb;17(11):36

6. Ferreira SAJ, Fernandes EC, Peixoto S. Como diagnosticar e tratar a osteoporose. Rev Bras Reumat.2004 dez;61(12):117-124.

7. Iwamoto J, Makita K, Sato Y, Takeda T, Matsumoto $\mathrm{H}$. Alendronate is more effective than elcatonin in improving pain and quality of life in postmenopausal women with osteoporosis. Osteoporos Int.2010 Nov 23.

8. Li WC, Chen YC, Yang RS, Tsauo JY. Effects of exercise programmes on quality of life in osteoporotic and osteopenic postmenopausal women: a systematic review and meta-analysis. Clin Rehabil. 2009 Oct; 23(10):888-96.

9. Aranha LLM, Mirom CJA, Alonso SM, Del PMJ, Sáenz GMC. Qualidade de vida relacionada à saúde em espanholas com osteoporose. Rev Saúde Pub.2006;40(2):298-303.

10. Sobrinho EAG, Carvalho MB, Franzi SA. Aspectos e tendências da avaliação da qualidade de vida de pacientes com câncer de cabeça e pescoço. Rev Soc Bras Cancer.2002 mai;15:14-22.

11. Baczyk G. Quality of life of women with osteoporosis - review of literature. Ortop Traumatol Rehabil. 2009 Jul-Aug;11(4):291-303. 
12. Ciconelli RM. Tradução para o Português e validação do questionário genérico de avaliação de qualidade de vida. 1997.120 f. Tese (Doutorado) Universidade Federal de São Paulo, São Paulo;1997.

13. Kastelan D, Vlak T, Lozo P, Gradiser M, Mijic S, Nikolic T, Miskic B, Car D, Tajsic G, Dusek T, Jajic Z, Grubisic F, Poljicanin T, Bakula M, Dzubur F, Strizak-Ujevic M, Kadojic M, Radman M, Vugrinec M, Kuster Z, Pekez M, Radovic E, Labar L, CrncevicOrlic Z, Korsic M. Health-related quality of life among patients with postmenopausal osteoporosis treated with weekly and monthly bisphosphonates. Endocr Res.2010;35(4):165-73.

14. Teixeira MJ. A lesão do trato de lissauer e do corno posterior da substância cinzenta da medula espinal e a estimulação elétrica do sistema nervoso central para o tratamento da dor por deseferentação. 1990. 256 f. Tese (Doutorado) - Faculdade de Medicina, Universidade de São Paulo, São Paulo;1990.

15. Lemos DCM, Miamoto TS, Valim V, Natour J. Qualidade de Vida em Pacientes com Osteoporose: Correlação entre OPAQ e SF-36. Rev Bras Reumat.2006 out;46(5):323-28.
16. Jovine SM, Buchalla MC, Santarém MME, Santarém MJ, Aldrighi MJ. et al. Efeito do treinamento resistido sobre a osteoporose após a menopausa: estudo de atualização. Rev Bras Epidem.2006;9(4):493-505.

17. Cantarelli BF, Simões JFM, Oliveira ML, Ferras BM, Szejnfeld LV. Qualidade de vida em pacientes com fraturas por osteoporose: adaptação cultural, reprodutibilidade e validação do "Osteoporosis Assessment Questionnaire-OPAQ". Rev Bras Reumat.1999 fev; 39(1):9-19.

18. Menduksheva IuE, Kirpikova MN, Shutemova EA. The influence of exercise therapy on the psychological status of women presenting with osteoporosis. Vopr Kurortol Fizioter Lech Fiz Kult. 2010 Jan-Feb;(1):23-5

19. Angin E, Erden Z. The effect of group exercise on postmenopausal osteoporosis and osteopenia. Acta Orthop Traumatol Turc. 2009 Aug-Oct;43(4):343-50.

20. Baczyk G. Quality of life of women with osteoporosis - review of literature.Ortop Traumatol Rehabil. 2009 Jul-Aug;11(4):291-303. 